\title{
Recomendaciones de la Sociedad Latinoamericana de Nefrología e Hipertensión para la atención ambulatoria nefrológica y en el primer nivel de atención durante la pandemia de COVID-19
}

\begin{abstract}
Alberto Alles ${ }^{1}$, Alfonso M. Cueto-Manzano ${ }^{2 *}$, Pablo G. Ríos-Sarro ${ }^{3}$, Adriana Robayo, Vicente Sánchez-Polo ${ }^{5}$, Ricardo Silvariño ${ }^{6}$, Laura Sola ${ }^{7}$, Augusto Vallejos ${ }^{8}$ y Carlos Zúñiga ${ }^{9}$

${ }^{1}$ Coordinación General de Comités, Sociedad Latinoamericana de Nefrología e Hipertensión, Rosario, Argentina; ${ }^{2}$ Unidad de Investigación Médica en Enfermedades Renales, Hospital de Especialidades, Centro Médico Nacional de Occidente, Instituto Mexicano del Seguro Social, Guadalajara, México; ${ }^{3}$ Comisión Asesora en Salud Renal de Uruguay, Montevideo, Uruguay; ${ }^{4}$ Dirección Ejecutiva del Instituto de Evaluación Tecnológica en Salud, Bogotá, Colombia; ${ }^{5}$ Servicio de Nefrología y Trasplante Renal, Hospital General de Enfermedades, Instituto Guatemalteco de Seguridad Social, Ciudad de Guatemala, Guatemala; ${ }^{6}$ Centro de Nefrología, Hospital de Clínicas, Facultad de Medicina, Universidad de la República, Montevideo, Uruguay; ${ }^{7}$ Centro de Hemodiálisis Crónica, Centro de Asistencia del Sindicato Médico del Uruguay, Institución de Asistencia Médica Privada de Profesionales sin Fines de Lucro, Montevideo, Uruguay; ${ }^{8}$ Coordinación Nacional del Programa de Abordaje Integral de Enfermedades Renales, Ministerio de Salud, Buenos Aires, Argentina; ${ }^{9}$ Servicio de Nefrología, Hospital Las Higueras, Talcahuano, Chile
\end{abstract}

\section{Resumen}

La situación de emergencia actual por COVID-19 ha originado una redistribución de los recursos humanos y materiales para la prevención y atención de pacientes con infección por el SARS-CoV-19, con la consecuente disminución de las acciones para la prevención y atención de los pacientes con enfermedad renal crónica (ERC), sobre todo en etapas avanzadas, o con otras afecciones crónicas y degenerativas. En este contexto, el Comité de Salud Renal de la Sociedad Latinoamericana de Nefrología e Hipertensión (SLANH) ha elaborado el siguiente documento con recomendaciones para la atención y continuidad del cuidado de pacientes con ERC en todas sus etapas, tanto por parte del equipo especialista en nefrología como por el equipo de atención primaria en salud. Ante una situación nueva como la pandemia actual, es muy probable que surjan modificaciones y actualizaciones frecuentes de las recomendaciones, las cuales se comunicarán a toda la comunidad nefrológica. Mientras tanto, este documento será de gran utilidad para proporcionar el mejor tratamiento posible para la ERC en la región.

Palabras clave: COVID-19. Enfermedad renal crónica. Cuidado nefrológico. Atención primaria de la salud.

\section{Recommendations of Latin American Society of Nephrology and Hypertension for the nephrological ambulatory and primary health-care attention during the COVID-19 pandemic}

\section{Abstract}

The current emergency situation due to COVID-19 has originated a redistribution of human and material resources for prevention and management of patients with SARS-CoV-19 infection, with the subsequent decrease in preventive and treatment actions for patients with chronic kidney disease (CKD) (particularly late stage), and/or other chronic

\section{Correspondencia:}

*Alfonso M. Cueto-Manzano

E-mail: a_cueto_manzano@hotmail.com

444-9032/O 2020 Sociedad Latinoamer licencia CC BY-NC-ND (http://creativecommons.org/licenses/by-nc-nd/4.0/)
Fecha de recepción: 29-06-2020

Fecha de aceptación: 14-08-2020

DOI: 10.24875/NEFRO.20000028
Disponible en internet: 17-12-2020

Nefro Latinoam. 2020;17:68-74 www.nefrologialatinoamericana.com 
non-communicable diseases. In this regard, the Kidney Health Committee of the Latin American Society of Nephrology and Hypertension (SLANH) has created this document with recommendations for the management and continuing care of patients with all stages of CKD, both by the Nephrology specialist team and by the primary health-care team. Facing the new situation with the pandemic by COVID-19 is highly probable that frequent modification and update of the present recommendations emerge, which in turn will be subsequently spread to the Nephrological community. Meanwhile, the expressed in this document would be of great help to provide the best possible CKD treatment in our region.

Key words: COVID-19. Chronic kidney disease. Nephrological care. Primary health-care.

\section{Introducción}

La pandemia por el COVID-19 ha golpeado con diferente intensidad a los países de Latinoamérica y obligado a redistribuir los recursos humanos y materiales en salud en favor de la prevención y la atención de los pacientes contagiados por el virus, en detrimento de las acciones relacionadas con la prevención, seguimiento y cuidado de personas con enfermedades crónicas.

Un ejemplo de ello son los pacientes con enfermedad renal crónica (ERC), en especial en etapas avanzadas, cuyos controles médicos, estudios de valoración y acceso a los medicamentos se han suspendido o postergado. Algunas publicaciones han considerado este retroceso en la continuidad del cuidado como un efecto colateral de la pandemia y sus efectos sanitarios serán evidentes en el corto plazo al aumentar la morbimortalidad vinculada con la ERC avanzada.

En este contexto, el Comité de Salud Renal de la Sociedad Latinoamericana de Nefrología e Hipertensión (SLANH) ha elaborado el siguiente documento con recomendaciones para la atención y continuidad del cuidado de pacientes con ERC en etapas 1 a 5 ( $\sin$ diálisis) durante la pandemia; el objetivo es mantener la calidad de la asistencia nefrológica.

Este documento utilizó como base las guías de práctica clínica internacionales y regionales, sin perder de vista en ningún momento las diferentes realidades sanitarias que viven los países de Latinoamérica.

La mayor parte de las recomendaciones carece de evidencia de calidad, por lo que no puede establecerse un "grado de evidencia" como sucede en otros documentos, como las guías de práctica clínica. Al momento de su publicación, estas recomendaciones son por tanto el resumen más actualizado. Es muy probable que surjan modificaciones frecuentes, las cuales se comunicarán a toda la comunidad nefrológica latinoamericana. Esta versión podrá mejorarse con los avances del conocimiento y con los aportes de la comunidad entera.

Recomendaciones para el equipo especialista en nefrología
1 Estrategias para mantener la continuidad y la calidad de la atención médica de los pacientes con ERC durante la pandemia por COVID-19 Recomendaciones generales

1. Suspender de forma transitoria las consultas, los estudios programados o las prácticas no esenciales, en los países y regiones donde la propagación del virus así lo exija.

2. Sustituir la consulta presencial programada, en la medida de lo posible, por otras alternativas, entre ellas la telemedicina (con cualquier medio disponible de las nuevas tecnologías de información y comunicación), la telefonía convencional o cualquier otro recurso de comunicación disponible en la institución.

3. Mantener una conducta activa en la programación de citas de los pacientes y destinar personal específico al llamado y concertación de las consultas para reducir al mínimo las pérdidas de seguimiento.

4. Mientras la consulta con el especialista no sea posible, se sugiere recomendar a los pacientes seguir con la medicación prescrita a dosis similares, incluidos los inhibidores de la enzima convertidora de angiotensina y los bloqueadores del receptor de angiotensina II, a menos que exista indicación por el médico tratante.

5. En la consulta telefónica debe realizarse un interrogatorio para identificar síntomas que sugieran infección por SARS-CoV-2 en curso.

6. Ante una probable infección se recomendará permanecer en el domicilio y contactarse con el prestador de salud para coordinar una valoración médica.

7. Siempre que sea posible en el país o la región deben programarse las extracciones de sangre en el domicilio del paciente.

8. Se recomendará en todos los casos probables NO POSTERGAR LAS PRUEBAS URGENTES en busca de infección por SARS-CoV-2. 
9. Deben explicarse a todos los pacientes los síntomas del COVID-19, así como los síntomas y signos de enfermedad renal que exigen una consulta urgente.

10. Conceder especial cuidado a los pacientes que reciben inmunosupresores (véase más adelante en este documento).

11. Se recomienda llevar un registro de la teleconsulta o la telefonía convencional en la historia clínica del paciente, con las acciones indicadas por el médico.

12. Es recomendable seguir una guía de valoración sistemática telefónica convencional 0 de teleconsulta.

13. Se sugiere asegurar la provisión de medicamentos en la farmacia de la institución. En los casos en que sea posible, el nefrólogo actualizará la totalidad de la medicación que recibe el paciente (nefrológica y no nefrológica) para confirmar la continuidad de la toma y reducir al mínimo el contacto del paciente con el sistema. Otras alternativas son la renovación automática de la medicación crónica o su provisión al domicilio de los pacientes, sobre todo de aquellos que reciben inmunosupresores.

14. Coordinar las diferentes áreas para ofrecer un abordaje interdisciplinario.

15. Debe promoverse que el nefrólogo tenga interconsultas programadas (telefónicas o por otros medios) con otros colegas y con personal de salud multidisciplinario para evitar desplazamientos innecesarios de los pacientes.

16. Asegurar la continuidad de la consulta con nutricionista y apoyo en salud mental. Para ello deben optimizarse canales de comunicación con los pacientes y sus familias a través de llamadas telefónicas o videollamadas. Pueden ser de gran ayuda los videos explicativos.

17. Se recomienda continuar con el programa de vacunaciones en la ERC: vacuna antiinfluenza, vacunación antineumocócica (13 y 23), y vacuna contra la hepatitis $B$.

18. Es fundamental no postergar la creación del acceso vascular definitivo para hemodiálisis o la colocación del catéter peritoneal para diálisis peritoneal, en caso que exista indicación para hacerlo. Debe considerarse una cirugía programada esencial.

19. En los pacientes que no requieran consulta presencial, en virtud de su situación clínica y etapas tempranas de la ERC, se recomienda comunicarse por los medios alternativos ya mencionados, reprogramar los estudios, asegurar el acceso a la medicación y agendar una próxima consulta acorde a la situación clínica y la emergencia sanitaria.

2 Recomendaciones para la consulta presencial

1. Todo personal del equipo de salud asignado a la tarea de atención sanitaria debe someterse a cribado de síntomas de COVID-19 antes de iniciar la jornada laboral.

2. En los pacientes en quienes se identifique una causa que justifique una consulta presencial, se recomienda coordinarla en el menor lapso de tiempo. La consulta presencial se coordinará en un día y un horario que reduzcan al mínimo el contacto con otros pacientes. Deben descartarse síntomas de COVID-19 por vía telefónica.

3. En todos los pacientes se recomiendan las siguientes medidas al ingresar al centro asistencial: pasar sobre un paño humedecido en hipoclorito, someterse a interrogatorio sobre síntomas de COVID-19, tomarse la temperatura, aplicarse alcohol o gel desinfectante en manos, exigir uso de tapabocas, barbijos o mascarillas protectoras y mantener una distancia recomendable de 1.5 a 2.0 m entre sí.

4. Los pacientes con síntomas indicativos de infección por COVID-19 se derivarán inmediatamente al lugar de atención específico designado para ellos mismos.

3 Recomendaciones para la atención ambulatoria de pacientes que reciben inmunosupresores

1. En pacientes estables, sin contacto con COVID-19 y asintomáticos, que reciben inmunosupresión de mantenimiento, se recomienda un seguimiento telefónico convencional o de teleconsulta para reforzar las medidas de aislamiento social y disipar las dudas. Si la enfermedad renal está estable, mantener la inmunosupresión sin cambios.

2. En el caso de pacientes con enfermedad nefrológica inicial, sin contacto con pacientes positivos a COVID-19 y asintomáticos, que reciben o deben recibir inmunosupresión de inducción, debe determinarse en primera instancia si existe riesgo vital y, si ése es el caso, mantener pautas de inmunosupresión.

3. Si no existe riesgo vital, se recomienda ajustar la inmunosupresión (fármaco, dosis) al riesgo de evolución de la enfermedad renal. En este último caso debe mantenerse seguimiento estrecho.

4. En casos sospechosos de COVID-19 bajo tratamiento inmunosupresor, NO POSTERGAR LA 
PRUEBA URGENTE en busca de infección por SARS-CoV-2.

5. En paciente con COVID-19 confirmado o probable, ya sea con aislamiento domiciliario u hospitalario, debe promoverse la consulta nefrológica para facilitar el control específico de la inmunosupresión y los episodios clínicos relacionados con ella, como apoyo al grupo asignado en el abordaje del COVID-19. Esta consultoría nefrológica debe hacerse preferentemente por teleconsulta.

6. En pacientes con infección respiratoria alta y PCR positiva a SARS-CoV-2 deben considerarse la suspensión o la disminución transitoria de los fármacos inmunosupresores.

Recomendaciones para el equipo de atención primaria de la salud al cuidado de pacientes con enfermedad renal crónica de etapas 4 o 5

\section{Referencia al nefrólogo}

En el contexto de esta pandemia, los pacientes con ERC que requieren atención médica evitan concurrir a los hospitales por el mayor riesgo de contagio y el exceso de demanda relacionado con el COVID-19. Su opción más cercana y factible de acceso al sistema de salud es acudir a la atención primaria en salud (APS). En consecuencia, es previsible que los equipos de atención primaria reciban a pacientes con ERC avanzada (etapas $4 \circ 5$ ), con múltiples comorbilidades, polifarmacia y síntomas complejos.

¿Cómo se define la enfermedad renal crónica?

La ERC se define como la presencia de alteraciones en la estructura o función renales durante al menos tres meses y con riesgos para la salud. Los criterios diagnósticos de la ERC incluyen a los denominados marcadores de daño renal (en particular la excreción urinaria de proteínas en cifras patológicas) o la reducción de la filtración glomerular (FG) $<60 \mathrm{ml} / \mathrm{min} / 1.73 \mathrm{~m}^{2}$ (Fig. 1).

¿Cómo optimizar la interacción de la atención primaria y su referencia a nefrología?

En condiciones habituales, sin emergencia epidemiológica, existe el consenso en las guías clínicas sobre tratamiento de la ERC de que los pacientes en las etapas 1 a 3b se controlen en la APS y aquéllos con ERC avanzada (etapas 4 y 5) se refieran al nefrólogo para estudio y tratamiento especializado, si bien algunos pacientes requieren la intervención del nefrólogo aun en las etapas primarias de la enfermedad (véase más adelante). El cumplimento de esta última recomendación está limitada en el contexto de la actual pandemia por COVID-19; por lo tanto, es prioritario disponer de vías de comunicación entre la APS y el nefrólogo de referencia. Esta

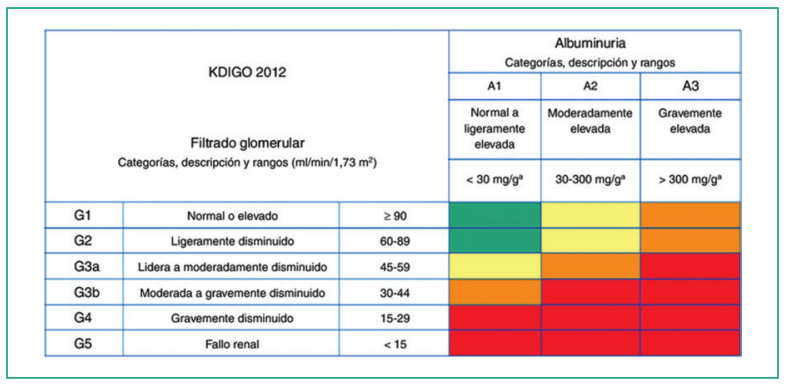

Figura 1. Etapas de la enfermedad renal crónica y riesgo de evolución de acuerdo con las categorías de filtración glomerular y albuminuria.

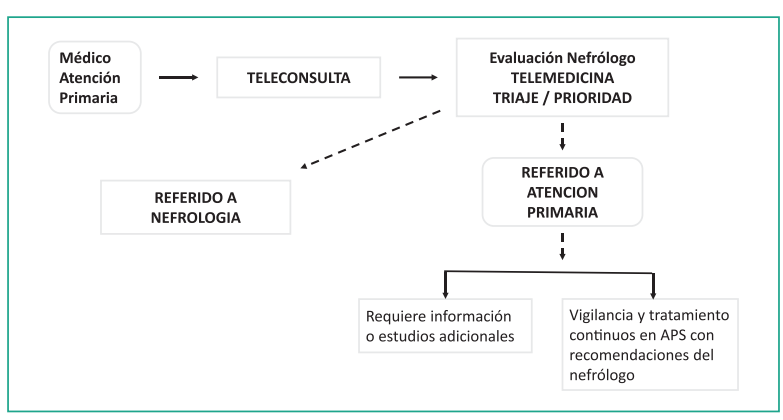

Figura 2. Flujograma propuesto de telenefrología.

interacción puede facilitarse con las nuevas tecnologías de información y comunicación utilizadas en la telemedicina (modalidad sincrónica o asincrónica), como videoconferencias, mensajería instantánea, correos electrónicos, transmisión de datos, llamadas telefónicas y plataformas en línea. En fecha reciente se ha demostrado que casi el $60 \%$ de las teleconsultas desde APS puede resolverlo el nefrólogo en línea. En consecuencia, es muy recomendable la utilización de cualquiera de estas tecnologías en la emergencia epidemiológica, si bien se reconoce que su empleo depende de la disponibilidad existente en cada país.

Se recomienda asimismo la creación de un formulario de registro, acotado y simple, que incluya antecedentes clínicos relevantes del paciente, motivo de la teleconsulta, estudios de laboratorio y tratamientos recibidos en la APS.

La Figura 2 muestra una propuesta de flujograma telemédico entre los dos niveles de atención, que puede ser sincrónica o asincrónica. Esta última modalidad no requiere que ambos profesionales estén disponibles de manera simultánea y acorta el tiempo/nefrólogo por consulta. El médico de APS emite su teleconsulta al nefrólogo interconsultor, quien valora los antecedentes clínicos y decide entre las siguientes opciones: 
Tabla 1. Criterios para solicitar interconsulta por telemedicina al nefrólogo durante la pandemia por COVID-19

- Pacientes con ERC en etapas 4 o 5 ( $\left.F G<30 \mathrm{ml} / \mathrm{min} / 1.73 \mathrm{~m}^{2}\right)$

- Sospecha de injuria renal aguda o crónica agudizada, descartado previamente de causas prerrenales

- Excreción urinaria de proteínas significativa y sostenida: cociente albúmina/creatinina $\geq 300 \mathrm{mg} / \mathrm{g}$ o cociente proteínas totales/creatinina $\geq 500 \mathrm{mg} / \mathrm{g}$ o proteinuria $\geq 500 \mathrm{mg} / 24 \mathrm{~h}$

- Avance de la ERC (descenso sostenido del $\mathrm{FG}>5 \mathrm{ml} / \mathrm{min} / 1.73 \mathrm{~m}^{2}$ al año)

- Hematuria no explicada por otras causas: $>20$ eritrocitos/ campo, con eritrocitos dismórficos $(30 \%)$ o cilindros hemáticos

- Pacientes con hipertensión arterial resistente (no controlada con una combinación de tres fármacos, incluido un diurético)

A) Contrarreferir al paciente a su unidad original cuando se requieren otros antecedentes o estudios, o bien reenviar con sugerencias o recomendaciones para continuar control y tratamiento en su centro de APS. En este tiempo de pandemia esto evitaría traslados innecesarios y reduciría el riesgo de contagio.

B) Enviar al centro de referencia para atención presencial del nefrólogo puesto que se necesitan estudios o tratamientos especializados. El médico de APS recibirá la respuesta del especialista mediante mensaje de texto, correo electrónico o llamada telefónica para su revisión y posterior inclusión en su ficha clínica.

\section{¿Cuándo derivar al nefrólogo?}

Para optimizar la escasa disponibilidad de citas para atención en nefrología es necesario tener siempre presentes las indicaciones para la derivación oportuna al especialista (Tabla 1). Para las citas presenciales tienen prioridad los pacientes en etapas 405 sin diálisis que, desde una estabilidad previa, sufran alguna descompensación o deterioro de la función renal, y por tanto requieran valoración presencial rápida. Los pacientes con síntomas graves (edema agudo pulmonar, hiperpotasemia grave, síndrome urémico, hipertensión maligna 0 acelerada) deben derivarse inmediatamente a un servicio de urgencias para su atención por nefrología.

2 Objetivos del tratamiento médico en pacientes con enfermedad renal crónica de etapas 4 o 5 que acuden a APS durante la pandemia por COVID-19

Los objetivos terapéuticos en las etapas 405 de la ERC son detener o retardar la evolución del daño renal, disminuir la morbimortalidad cardiovascular, identificar y tratar las complicaciones adjuntas (anemia, hipertensión arterial, alteraciones osteometabólicas, hiperuricemia, acidosis, entre otras) y dar tiempo para que el paciente elija en forma informada y programada por algún tratamiento de reemplazo renal: hemodiálisis/peritoneodiálisis, trasplante renal o tratamiento conservador no dialítico.

\section{¿Cómo debe tratarse la hipertensión arterial?}

Además del control del sodio de la dieta, deben considerarse los fármacos betabloqueadores, antagonistas del calcio y diuréticos. Los inhibidores de la enzima convertidora de angiotensina (IECA) y los antagonistas del receptor de angiotensina (ARA2) son de elección en pacientes con hipertensión y proteinuria. La mayoría de los pacientes necesita dos o más fármacos antihipertensivos (uno de ellos un diurético, de preferencia de asa). El objetivo de presión debe relacionarse con la proteinuria en diabéticos y no diabéticos: objetivo $<130 / 80 \mathrm{mmHg}$ en pacientes con cociente albúmina/ creatinina $\geq 30 \mathrm{mg} / \mathrm{g} ;<140 / 90 \mathrm{mmHg}$ en enfermos sin albuminuria. El descenso de la presión arterial debe ser gradual, sobre todo en ancianos, y debe evitarse la hipotensión ortostática.

\section{¿Cuál es el objetivo en la proteinuria?}

Además de las medidas nutricionales, que incluyen ajuste de proteínas y sodio en la dieta, para el control de la proteinuria se sugiere utilizar IECA/ARA2. Dado el riesgo de hiperpotasemia en las fases avanzadas de la ERC, estos fármacos se deben administrar con vigilancia periódica de los valores de potasio. El objetivo es lograr el mayor descenso posible y alcanzar al menos un cociente proteínas/creatinina $<500 \mathrm{mg} / \mathrm{g}$ o proteinuria $<500 \mathrm{mg} / 24 \mathrm{~h}$.

\section{¿Cómo debe tratarse la hiperglucemia?}

La hiperglucemia sostenida se relaciona con un mayor deterioro de la función renal y evolución a la falla renal extrema. La evaluación del control de los pacientes diabéticos con ERC es similar a la de aquéllos sin ERC, pero en las etapas más avanzadas, sobre todo con múltiples comorbilidades o mayor riesgo de hipoglucemia, se recomienda un objetivo de $\mathrm{HbA}_{1 \mathrm{C}}<8 \%$, siempre en relación con las características particulares de cada paciente. Debe ajustarse la dosis de varios antidiabéticos orales en pacientes con ERC avanzada, pero en particular se recomienda evitar el uso de metformina en estadios 405 , o desde el estadio 3 si no hay signos de estabilidad de la función renal.

\section{¿Tiene utilidad mejorar el perfil de lípidos?}

La dislipidemia se acompaña de mayor deterioro de la función renal y avance de la enfermedad, además de incrementar el riesgo cardiovascular; por lo tanto, el control lipídico es muy importante. Sin embargo, 
debido al riesgo de efectos colaterales indeseables e interacción farmacológica, se recomienda reducir $50 \%$ la dosis de estatinas (excepto atorvastatina y pravastatina) en pacientes con ERC de fase 405.

¿Cuáles son las recomendaciones farmacológicas para tratar el dolor de la ERC avanzada?

Los fármacos para aliviar el dolor en pacientes con ERC avanzada son habitualmente los mismos prescritos en la población general, pero requieren en ocasiones ajuste posológico. Como regla general, se recomienda evitar su uso irracional o injustificado en pacientes con enfermedad renal avanzada. Es recomendable reducir al $75 \%$ la dosis normal si el índice de FG se halla entre 10 y $50 \mathrm{ml} / \mathrm{min}$, y al $50 \%$ de la dosis normal si es $<10 \mathrm{ml} / \mathrm{min}$.

¿Qué otras enfermedades crónicas pueden tratarse en consulta y bajo supervisión del nefrólogo?

Otras anomalías o complicaciones de la ERC, como anemia, alteraciones del metabolismo mineral y óseo, acidosis o trastornos hidroelectrolíticos, en virtud de su complejidad y multiplicidad de variables determinantes, pueden abordarse durante la teleconsulta para establecer un plan de atención en la APS durante la pandemia.

3 Requerimientos nutricionales que deben considerarse en la enfermedad renal crónica de etapas 4 o 5 aún sin diálisis

Algunas recomendaciones generales de nutrición que pueden consultarse por telemedicina con el nefrólogo de referencia son las siguientes:

¿Cuál es el aporte de calorías/día que requiere el paciente con ERC de etapas 405 ?

En las etapas 4 o 5 se recomiendan $30-35 \mathrm{kcal} / \mathrm{kg}$ de peso ideal. El requerimiento calórico puede aumentar o disminuir según sea el estado nutricional (obesidad o desnutrición).

¿Cuál debe ser el aporte proteico en las etapas 4 o 5 de la ERC?

La disminución de proteínas de la dieta tiene como objetivo reducir la evolución de la ERC. El aporte proteico recomendado en la ERC en etapas 405 es de $0.8 \mathrm{~g} / \mathrm{kg}$ peso ideal, pero es preciso tener sumo cuidado para no desarrollar desnutrición o acentuarla. Una restricción proteica mayor debe consensuarse con el equipo nefrológico referencial.

En cuanto al fósforo, ¿cuál debe ser el aporte diario?

La hiperfosfatemia se relaciona con morbimortalidad cardiovascular. En etapas avanzadas de la ERC se recomienda restringir el aporte de fósforo a $800 \mathrm{mg} /$ día. El fósforo está presente en concentraciones elevadas en las carnes rojas, lácteos, semillas y proteínas de origen animal.

En cuanto al sodio de la dieta, ¿su uso debe individualizarse $o$ es igual para todo enfermo renal?

Se recomienda restringir el consumo de sodio a $1.2 \mathrm{~g} /$ día (equivale a $<3.0 \mathrm{~g}$ de sal común) en todas las etapas de la ERC. Aunque la adherencia a una dieta así es más difícil de lograr, se recomienda estimular el apego para lograr reducciones efectivas de la presión arterial y el riesgo de insuficiencia cardíaca. En las regiones afectadas por la nefropatía mesoamericana, los pacientes no deben limitar el sodio y la dieta debe ser normosódica.

¿Cuál es el aporte de potasio en las etapas 40

\section{5 de la ERC?}

Las recomendaciones para la restricción de potasio han cambiado en fecha reciente y ahora se prioriza la individualización de los casos y se acepta un mayor consumo hasta de 3,000 mg/día. Se recomienda vigilar el potasio con frecuencia.

¿Qué cantidad de líquidos pueden ingerir los pacientes?

El aporte de líquidos en etapa prediálisis no debe restringirse en la mayoría de los casos, por lo que puede utilizarse la recomendación de personas sanas de 30-35 ml/kg. Los pacientes con cardiopatía congestiva o edema generalizado deben limitar la sal y los líquidos de modo individual y derivarse a la valoración del nefrólogo con prioridad.

Durante la pandemia por COVID-19, en todos los pacientes con ERC, debe promoverse en la APS

El estilo de vida saludable, con el mantenimiento de un peso adecuado, consumo de una dieta saludable y ajustada a la etapa de la ERC, realizar ejercicio dentro de las capacidades y estado físico de los pacientes y evitar las toxicomanías. Las medidas de sano distanciamiento, higiene de manos y otras aplicables a la población general se recomiendan también en los enfermos renales.

\section{Agradecimientos}

Los autores agradecen a la Sociedad Latinoamericana de Nefrología e Hipertensión.

\section{Conflicto de intereses}

Los autores manifiestan no tener conflicto de intereses. 


\section{Responsabilidades éticas}

Protección de personas y animales. Los autores declaran que para esta investigación no se han realizado experimentos en seres humanos ni en animales.

Confidencialidad de los datos. Los autores declaran que en este artículo no aparecen datos de pacientes.

Derecho a la privacidad y consentimiento informado. Los autores declaran que en este artículo no aparecen datos de pacientes.

\section{Bibliografía}

- Silvariño R, Boggia J, Seija M, Baccino C, San Román S, Luzardo L, et al. Recomendaciones para la actuación clínica y organización asistencial durante la pandemia por SARS-CoV-2 en nefrología. Rev Méd Urug. 2020;36 (2):219-238.
- Osman MA, Alrukhaimi M, Ashuntantang GE, Bellorin-Font E, Benghanem Gharbi E, Braam B, et al. Global nephrology workforce: gaps and opportunities toward a sustainable kidney care system. Kidney Int Suppl. (2011) 2018;8(2):52-63.

- Gorostidi M, Santamaría R, Alcázar R, Fernández-Fresnedo G, Galcerán JM, Goicoechea M, et al. Documento de la Sociedad Española de Nefrología sobre las guías KDIGO para la evaluación y el tratamiento de la enfermedad renal crónica. Nefrología. 2014;34(3):302-316.

- Guías Latinoamericanas de Práctica Clínica Sobre la Prevención, Diagnóstico y Tratamiento de la Enfermedad Renal Crónica. Disponible en: https:// slanh.net/guias-latinoamericas-de-practica-clinica-sobre-la-prevenciondiagnostico-y-tratamiento-de-los-estadios-1-5-de-la-enfermedad-renal-cronica/

- Kidney Disease: Improving Global Outcomes (KDIGO) CKD Work Group. KDIGO 2012 Clinical Practice Guideline for the Evaluation and Management of Chronic Kidney Disease. Kidney Int Suppl. 2013;3:1-150.

- Curso virtual "Prevención y manejo de la enfermedad renal crónica para equipos del primer nivel de atención". Coorganizado por la Organización Panamericana de la Salud (OPS) y la Sociedad Latinoamericana de Nefrología e Hipertensión (SLANH). Disponible en: https://www.campusvirtualsp.org/es/curso/prevencion-y-manejo-de-la-enfermedad-renal-cronica-para-equipos-del-primer-nivel-de-atencion.

- $\quad$ Zuniga C, Riquelme C, Muller H, Vergara G, Astorga C, Espinoza M. Using telenephrology to improve access to nephrologist and global kidney management of CKD primary care patients. Kidney Int Rep. 2020;5(6):920-923. 